\title{
Intrathecal Baclofen Facilitated Postanesthetic Tracheal Extubation in a Dystonic Patient Associated with Neurodegeneration of Brain Iron Accumulation (Hallervorden-Spatz Disease)
}

\author{
Chien-Ching Lee, Ya-Chun Chu, Chia-Chun Chuang, Chih-Yang Chen, Mei-Yung Tsou*, \\ Kwok-Hon Chan \\ Department of Anesthesiology, Taipei Veterans General Hospital and School of Medicine, National Yang-Ming University, Shipai, \\ Chinese Taipei. \\ E-mail: otzison@hotmail.com
}

Received October $3^{\text {rd }}, 2011$; revised November $13^{\text {th }}, 2011$; accepted November $26^{\text {th }}, 2011$.

\begin{abstract}
Patients with Hallervorden-Spatz disease may be confronted by invasive procedure, like gastrostomy and thalamotomy for care of the status of extreme dystonia and rigidity. This rare disorder possesses potential perioperative risks, such as difficult airway management, aspiration pneumonia, hyperpyrexia, dehydration, acute renal failure, and postoperative pulmonary insufficiency. As patients were usually in the state of uncontrollable dystonia and rigidity, delayed endotracheal extubation had been suggested by several previous case reports. We report the anesthetic management of a 34-year-old Hallervorden-Spatz disease woman scheduled for intrathecal baclofen pump implantation under general anesthesia. Preoperatively she had suffered from severe dystonia, confusion and repeated attacks of respiratory insufficiency and pneumonia requiring mechanical ventilation and critical care. Anesthesia was induced uneventfully and the catheter of the pump reached the CSF via $T_{2}$ interspace. Intraoperative intrathecal baclofen injection mildly relieved her severe dystonia and rigidity. Hemodynamics and arterial blood gas were improved than preoperative. She was extubated after consciousness was regained. Postanesthetic visit demonstrated a fair outcome. Whether this treatment applies to every case confronted by general anesthesia needs to be elucidated. Nevertheless, we suggest intrathecal baclofen may help postanesthetic pulmonary care by attenuating dystonia and rigidity and can be an adjuvant for anesthesia care in patients with Hallervorden-Spatz disease.
\end{abstract}

Keywords: Anesthesia, Hallervorden-Spatz Disease, Intrathecal, Baclofen, Pneumonia

\section{Introduction}

Hallervorden-Spatz disease (HSD) is a rare disorder characterized by movement abnormalities and dementia. [1] Its etiology is related to neurodegeneration with iron accumulation in the brain [1]. Rapid progression of the disease may lead to severe disability, life-threatening state, the status dystonicus, and several critical events such as hyperpyrexia, dehydration, respiratory insufficiency and acute renal failure secondary rhabomyolysis $[2,3]$. Literature reviews demonstrated patients with HSD tend to suffer perianesthetic complications, ex. Involuntary movement and seizure upon noxious stimulation, difficult airway management, risk of aspiration and postanesthetic pulmonary disability [3]. Patients requireing general anesthesia for these invasive procedures were usually in a status of uncontrolled dystonia and rigidity that threatened their lives. Previous case reports suggested delayed tracheal extubation and prolonged intensive care after anesthesia $[4,5]$. Here we report a case of HSD with late onset and rapid progression. She suffered from extreme dystonia and rigidity and repeated attacks of respiratory insufficiency and pneumonia. Intrathecal baclofen pump implantation under general anesthesia was scheduled to relieve her severe dystonia. Intraoperative administration of baclofen helped relieve the dystonia and rigidity and tracheal extubation can be achieved earlier than usual. Postanesthetic visit also showed fa- 
vorable pulmonary outcome.

\section{Case Report}

A 34-year-old woman was scheduled for intrathecal baclofen pump insertion (SynchroMed ${ }^{\circledR}$ II programmable infusion pump) for controlling status dystonicus. Her past history can be traced back to about 4 years ago when symptoms, like involuntary head nodding, left hand tremor, and stiffness of lower extremity developed and gradually limited her activity. Her condition rapidly deteriorated. Severe dystonia and rigidity resulted in dystonic posturing, extreme rotation of the neck and head and voluntary movements impossible. Oromandibular rigidity made articulation and swallowing difficult and she was fed with a nasogastric tube. Mental deterioration was also noted recently. Brain $\mathrm{T}_{2}$-weighted Magnetic resonance imaging (MRI) displayed the typical appearance of "eyes of the tiger" in the globus pallidus (Figure 1) and hence diagnosed with HSD. She had received medical trial treatments including tetrabenazine, biperiden, risperidone, clozapine, baclofen, valproic acid, clonazepam and midazolam and surgeries for deep brain stimulation under local anesthesia but was unsuccessful. In the past years, she was admitted 4 times for respiratory insufficiency and pneumonia, and treated with mechanical ventilation, intravenous antibiotics and critical care. As the rapid progression of the disease, she was arranged for the intrathecal baclofen pump implantation under general anesthesia.

Preoperative hematological, biochemical tests, Electrocardiography (EKG) and Chest X-ray (CXR) were normal. In the operative room, standard monitorings including pulse oximetry, noninvasive blood pressure measurement, EKG, and capnography were established. Preoperatively the patient exhibited tachypnea (respiretory rate (RR): 24 breaths per minute) and tachycardia (heart rate (HR): 109 beats per minute). Before induction of anesthesia, an arterial catheter was inserted. Arterial blood gas $(\mathrm{ABG})$ showed moderate oxygenation $\left(\mathrm{PaO}_{2}=\right.$ $65 \mathrm{mmHg}$ on room air). After sufficient preoxygenation, anesthesia was induced with fentanyl $3 \mu \mathrm{g} / \mathrm{kg}$, propofol $2.5 \mathrm{mg} / \mathrm{kg}$, and rocuronium $1 \mathrm{mg} / \mathrm{kg}$. After the anesthestics were injected, dystonia and rigidity resolved and trachea was intubated without difficulty. Anesthesia was maintained with sevoflurane of end-tidal concentration within $2 \sim 3 \%$. For pump implantation, the catheter entered the cerebrospinal fluid (CSF) at $\mathrm{T}_{9}$ interspace and advancing to the level of $\mathrm{T}_{2}$, and the port was implanted below the costal margin. A bolus of baclofen $100 \mu \mathrm{g}$ was given immediately and the pump was programmed at 100 $\mu \mathrm{g} /$ day. Then spontaneous breathing returned after sevoflurane was discontinued and the patient was sent to

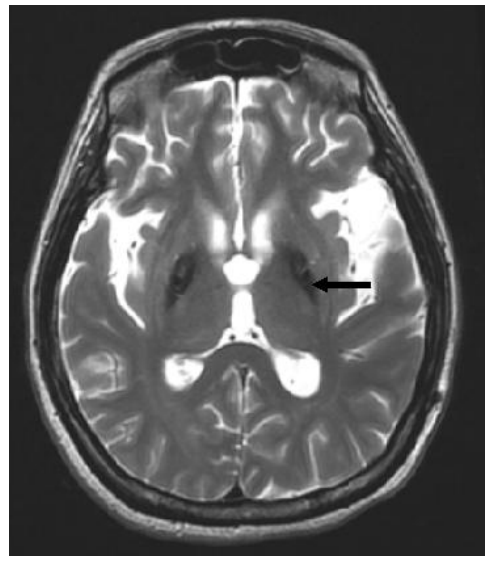

Figure 1. A typical $T_{2}$-weighted magnetic resonance image displaying bilaterally symmetric hyperintense signal changes in the anterior medial globus pallidus along with surrounding hypointensity in the globus pallidus. Black arrow indicates the typical "eye-of-the-tiger" sign.

postanesthetic care unit with endotracheal tube still inplace.

In the postanesthetic care unit, the patient awoke after half an hour. Endotracheal tube was removed for stable hemodynamics $(\mathrm{RR}=16, \mathrm{HR}=89)$ and fair $\mathrm{ABG}$ : $\mathrm{pH}$ 7.38, $\mathrm{PaO}_{2} 93 \mathrm{mmHg}$ and $\mathrm{PaCO}_{2} 40.0 \mathrm{mmHg}$ (room air). During the 4-h observational period, dystonia came back but was mildly relieved than that preoperatively. Pulse oximetry remained $99 \%$ saturation with room air and then she was sent back to ward. Postanesthetic visit on the following day revealed improved dystonia and rigidity, fair pulse oximetry and no fever. Chest auscultation showed clear breathing sounds. She was discharged 5 days after the surgery. We returned visit to check the stability of the implantation one week later after he discharged. Involuntary head nodding, left hand tremor, stiffness of lower extremity and oromandibular rigidity which made articulation and swallowing difficult are mildly relieved after intrathecal baclofen infusion pump.

\section{Discussion}

HSD is a rare, inherited, autosomal recessive neurodegenerative disorder associated with iron accumulation in the basal ganglion of the human brain [6-8]. The onset can be in children, adolescent, and adults and maybe familial or sporadic [6-8]. The exact pathophysiological mechanism of the disease is not yet clear. Mutation in a novel pantothenate kinate 2 gene (PANK2) has been found to be responsible for HSD [7,8]. This mutation lead to accumulation of cysteine and cysteine-containing compounds in the basal ganglia, consequently causing chelation of iron in the globus pallidus. Brain MRI revealed decreased signal intensity in both basal ganglion 
and small areas of hyperintensity in the segments on $T_{2}$ weighted images ("eye of the tiger" sign) $[9,10] . T_{2}$ weighted scans use a gradient echo sequence. The gradient echo sequence used does not have the extra refocusing pulse used in spin echo so it is subject to additional losses above the normal $T_{2}$ decay (referred to as $T_{2}{ }^{\prime}$ ). This also makes it more prone to susceptibility losses at air/tissue boundaries, but can increase contrast for certain types of tissue, such as venous blood.

Patients request for general anesthesia for invasive procedure are usually in a condition of status dystonica or dystonic storm $[2,11]$. This state is life-threatening and often requires intensive care. Dystonic pain may lead to rhabdomyolysis and renal failure. Pulmonary disability includes risk of aspiration and respiratory depression on the treatment of sedatives for the control of dystonia. Hyperpyrexia and dehydration are common [2,11]. Thus patients confront by certain anesthesia-related complications [3-5]. Progressive rigidity, especially oromandibular rigidity, difficulty in opening the mouth or trismus, may restrict mask ventilation and endotracheal intubation. Based on limited case reports, most dsytonia get relieved after intravenous anesthetics and the airway can be maintained. Propofol, thiopental, fentanyl, remifentanil, $\mathrm{N}_{2} \mathrm{O}$, volatile anesthetics and several neuromuscular blocking agents had been used without adverse effect $[4$, 5,12-14].

In 2000, Keegan et al. [5] reported a complicated case of an 11-year-old girl who received general anesthesia for stereotactic thalamotomy for her severe dystonia. In the postoperative care unit, acute respiratory insufficiency (airway obstruction) developed and an emergent endotracheal reintubation was performed. For most case reports, dystonia came back postoperatively after the anesthetics wore off. We are not sure whether surgical stress would exacerbate the muscle spasms, however, most reports suggested a delayed tracheal extubation after anesthesia. For our patient, preoperatively she exhibited a picture of near status dystonicus and impending respiratory insufficiency (despite of oral baclofen and midazolam previously), postoperative mechanical ventilation were scheduled. However, after she regained her consciousness, $\mathrm{ABG}$ also demonstrated fair condition and then she was extubated earlier than expected. This may not be the maximal or steady effect of the baclofen, however, together with the residual effect of anesthetics, they reduced the severity of dystonia and improved postoperative respiratory ability.

Baclofen is a derivative of gamma-aminobutyric acid (GABA). It is primarily used to decrease spasticity related to multiple sclerosis, spinal cord injuries, or other neurological diseases. Recently, intrathecal baclofen, to- gether with deep brain stimulation of globus pallidus internus proved to be effective in treating status dystonicus $[2,11]$. Our patient received a bolus of intrathecal baclofen intraoperatively and benefited in postoperative period without obvious delayed regain of consciousness. Other side effects of baclofen, such as dizziness, drowsiness, headaches, nausea, and weakness, may be hard to assess in this population of patient.

In conclusion, our patient received an intraoperative intrathecal baclofen which improved her status dystonicus postoperatively. This improvement helped for earlier tracheal extubation and a fair pulmonary outcome without delayed regain of consciousness in the postaensthetic visit. Therefore, we suggested intrathecal baclofen an adjuvant for anesthetic care in patients with HSD requiring general anesthesia in the future.

\section{REFERENCES}

[1] K. F. Swaiman, "Hallervorden-Spatz Syndrome," Pediatric Neurology, Vol. 25, No. 2, 2001, pp. 102-108. doi:10.1016/S0887-8994(01)00253-3

[2] M. Kyriagis, P. Grattan-Smith, A. Scheinberg, C. Teo, N. Nakaji and M. Waugh, "Status Dystonicus and Hallervorden-Spatz Disease: Treatment with Intrathecal Baclofen and Pallidotomy," Journal of Paediatrics Child Health, Vol. 40, No. 5-6, 2004, pp. 322-325. doi:10.1111/j.1440-1754.2004.00374.x

[3] J. Hinkelbeim, A. Kalenka and M. Alb, "Anesthesia for Patients with Pantothenate-Kinase-Associated Neurodegeneration (Hallervorden-Spatz Disease)-A Literature Review," Acta Neuropsychiatrica, Vol. 18, No. 3-4, 2006, pp. 168-172. doi:10.1111/j.1601-5215.2006.00144.x

[4] R. C. Roy, S. McLain, A. Wise and L. D. Shaffner, "Anesthetic Management of a Patient with HallervordenSpatz Disease," Anesthesiology, Vol. 58, 1983, pp. 382384. doi:10.1097/00000542-198304000-00017

[5] M. T. Keegan, R. P. Flick, J. Y. Matsumoto, D. H. Davis and W. L. Lanier, "Anesthetic Management for TwoStage Computer-Assisted, Stereotactic Thalamotomy in a Child with Hallervorden-Spatz Disease," Journal of Neurosurgical Anesthesiology, Vol. 12, No. 2, 2000, pp. 107-111. doi:10.1097/00008506-200004000-00006

[6] W. Halliday, "The Nosology of Hallervorden-Spatz Disease," Journal of the Neurological Sciences, Vol. 134, Supplement, 1995, pp. 84-91. doi:10.1016/0022-510X(95)00212-K

[7] S. J. Hayflick, S. K. Westaway, B. Levinson, B. Zhou, M. A. Johnson, K. H. Ching and J. Gitschier, "Genetic, Clinical, and Radiographic Delineation of HallervordenSpatz Syndrome," The New England Journal of Medicne, Vol. 348, 2003, pp. 33-40. doi:10.1056/NEJMoa020817

[8] S. Kapoor, K. Hortnagel, S. Gogia, R. Paul, V. Malhotra and A. Prakash, "Pantothenate Kinase Associated Neurodegeneration (Hallervorden-Spatz Syndrome)," Indian 
Journal of Pediatrics, Vol. 72, 2005, pp. 261-263.

[9] R. P. Guillerman, "The Eye-of-the-Tiger Sign," Radiology, Vol. 217, 2000, pp. 895-896.

[10] K. D. Sethi, R. J. Adams, D. W. Loring and T. El Gammal, "Hallervorden-Spatz Syndrome: Clinical and Magnetic Resonance Imaging Correlations," Annals of Neurology, Vol. 24, No. 5, 1988, pp. 692-694. doi:10.1002/ana.410240519

[11] P. Mariotti, A. Fasano, M. F. Contarino, G. D. Marca, M. Piastra, O. Genovese, S. Pulitanò, A. Chiaretti and A. R. Bentivoglio, "Management of Status Dystonicus: Our Experience and Review of the Literature," Movement Disorders, Vol. 22, No. 7, 2007, pp. 963-968. doi: $10.1002 / \mathrm{mds} .21471$

[12] E. C. Sheehy, P. Longhurst, D. Pool and M. Dandekar,
"Self-Inflicted Injury in a Case of Hallervorden-Spatz Disease," International Journal of Paediatric Dentistry, Vol. 9, No. 4, 1999, pp. 299-302. doi:10.1111/j.1365-263X.1999.00149.x

[13] I. Balas, N. Kovacs and K. Hollody, "Staged Bilateral Stereotactic Pallidothalamotomy for Life-Threatening Dystonia in a Child with Hallervorden-Spatz Disease," Movement Disorders, Vol. 21, No. 1, 2006, pp. 82-85. doi: $10.1002 / \mathrm{mds} .20655$

[14] P. Hurtado, L. Salvador, E. Carrero, J. Rumia and N. Fabregas, "Anesthesia Considerations for Deep-Brain Stimulation in a Patient with Type-2 Pantothenate Kinase Deficiency (Hallervorden-Spatz Disease)," Revista Española de Anestesiología y Reanimación, Vol. 56, No. 3, 2009, pp. 180-184. 